\title{
FEBRILE CONVULSIONS
}

\author{
H B Valman
}

\section{Definition}

\section{Characteristics of simple febrile convulsions}

- Last $<20$ minutes

- No focal features

- Occur in children aged 6 months to 5 years

- Occur in children with no development or neurological abnormalities

- Not repeated in the same episode

- Complete recovery within 1 hour

\section{Emergency treatment}

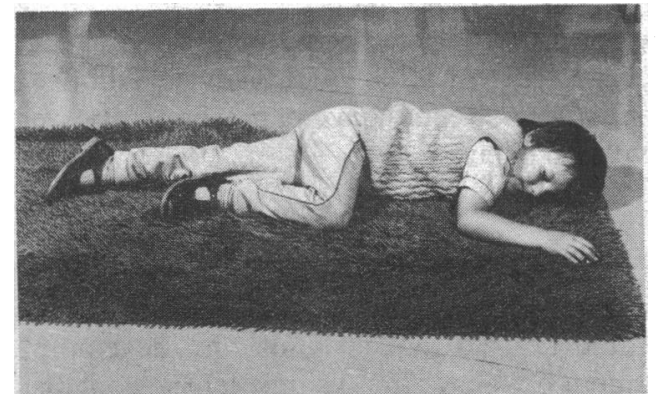

Nursing position to avoid vomiting with aspiration.

Rectal diazepam is the anticonvulsant of choice
A febrile convulsion is a fit occurring in a child aged from 6 months to 5 years, precipitated by fever arising from infection outside the nervous system in a child who is otherwise neurologically normal.

Convulsions with fever include any convulsion in a child of any age with fever of any cause. Among children who have convulsions with fever are those with pyogenic or viral meningitis, encephalitis, or cerebral palsy with intercurrent infections. Children who have a prolonged fit or who have not completely recovered within one hour should be suspected of having one of these conditions.

Most of the fits that occur between the ages of 6 months and 5 years are simple febrile convulsions and have an excellent prognosis. If there is no fever the possibility of epilepsy should be considered.

Often fever is recognised only when a convulsion has already occurred. An abrupt rise in temperature rather than a high level is important. There may be a frightened cry followed by abrupt loss of consciousness with muscular rigidity, which form the tonic stage. Cessation of respiratory movements and incontinence of urine and faeces may occur during this stage, which usually lasts up to half a minute. The clonic stage which follows consists of repetitive movements of the limbs or face. By arbitrary definition, in simple febrile convulsions the fit lasts less than 20 minutes, there are no focal features, and the child is aged between 6 months and 5 years and has been developing normally.

Rigors may occur in any acute febrile illness, but there is no loss of consciousness.

If the child has fever all his clothes should be removed and he should be covered with a sheet only. The child should be nursed on his side or prone with his head to one side because vomiting with aspiration is a constant hazard.

Rectal diazepam $(0.5 \mathrm{mg} / \mathrm{kg})$ produces an effective blood concentration of anticonvulsant within 10 minutes. The most convenient preparation resembles a toothpaste tube (Stesolid). Early admission to hospital or transfer to the intensive care unit should be considered if a second dose of anticonvulsant is needed.

All children who have had a first febrile convulsion should be admitted to hospital to exclude meningitis and to educate the parents, as many fear that their child is dying during the fit. Physical examination at this stage usually does not show a cause for the fever, but a specimen of urine should be examined in the laboratory to exclude infection and glucose stick testing should be performed. Most of these children have a generalised viral infection with viraemia. A febrile convulsion may occur in roseola at the onset and three days later the rash appears. Occasionally acute otitis media is present, in which case an antibiotic is indicated, but most children with febrile convulsions do not need an antibiotic. 


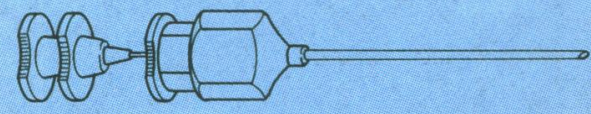

\section{Treatment}

- Paracetamol is the preferred drug for fever

- Rectal diazepam should be used as soon as possible after the onset of a convulsion

- Phenobarbitone should be used only for frequent recurrences

\section{Immunisation}

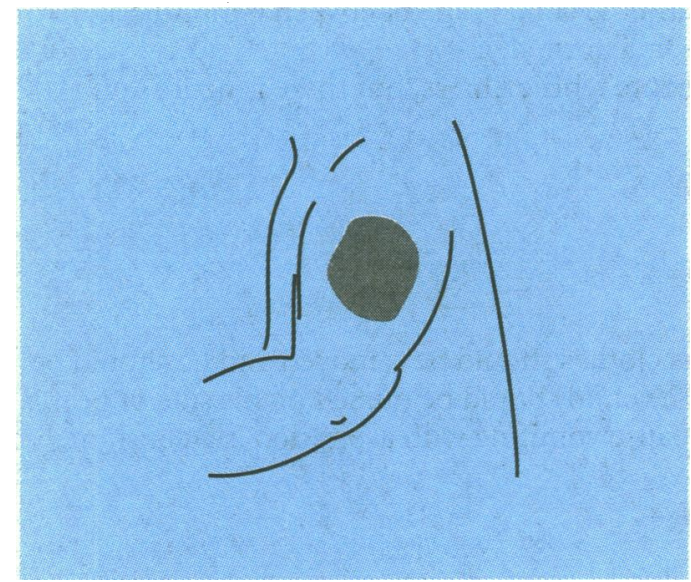

\section{Prognosis}

- The risk of subsequent epilepsy is small (about 1\%)

- Less than a third of children have further febrile convulsions

\section{Lumbar puncture}

A lumbar puncture should be performed if the child is under 18 months old or any of the following are present:

(1) Signs of meningism such as neck stiffness.

(2) Drowsiness, irritability, or systemic illness.

(3) Complex convulsion that contains any feature that does not conform with the definition of a simple convulsion.

Ideally the decision should be taken by an experienced doctor, who may decide on clinical grounds that lumbar puncture is unnecessary even in a younger child, but when in doubt the investigation should be performed. The doctor deciding not to undertake a lumbar puncture should review the patient personally within a few hours.

A child who has had severe vomiting or is in coma must be examined by an experienced doctor before lumbar puncture because of the risk of coning.

An electroencephalogram is not a guide to diagnosis, treatment, or prognosis.

\section{Management of fever}

There is no evidence that antipyretic treatment influences the recurrence of febrile convulsions, but fever should be treated to promote the comfort of the child and to prevent dehydration. The child's clothes should be taken off and he or she should be covered with a sheet only. Paracetamol is the preferred antipyretic, and adequate fluid should be given.

\section{Anticonvulsant drugs}

Rectal diazepam should be used as soon as possible after the onset of the convulsion. The parents should be advised not to give it if the convulsion has stopped.

The indications for long term anticonvulsant prophylaxis have changed and the sole indication-frequent recurrences, which should be treated with phenobarbitone-is rare. There is no evidence that in the minority of children who later develop epilepsy the prophylactic use of anticonvulsant drugs would have prevented it.

As immunisation against diphtheria, tetanus, pertussis, and poliomyelitis is given to children $2-4$ months old, this schedule is usually completed before febrile convulsions occur. Babies having convulsions with fever aged under 4 months should be assessed by a paediatrician. Children who have febrile convulsions before immunisation against diphtheria, pertussis, and tetanus because the immunisation has been delayed should be immunised after their parents have been instructed about the management of fever and the use of rectal diazepam.

Measles, mumps, and rubella immunisation should be given as usual to children who have had febrile convulsions, with advice about the management of fever to the parents. Rectal diazepam should be made available for use should a convulsion occur.

Unless there is clinical doubt about the child's current developmental or neorological state, parents should be told that prognosis for development is excellent. The risk of subsequent epilepsy after a single febrile convulsion with no complex features is about $1 \%$. With each additional complex feature the risk rises to nearly $50 \%$ in children with three complex features by the age of 25 years. Only about $1 \%$ of children with febrile convulsions are in this group.

The risk of having further febrile convulsions is about $30 \%$. This risk increases in younger infants and is about $50 \%$ in infants aged under 1 year at the time of their first convulsion. A history of febrile convulsions in a first degree relative is also associated with a risk of recurrence of about $50 \%$. A complex convulsion or a family history of epilepsy is probably associated with an increase in the risk of further febrile convulsions. 


\section{Information for parents}

Information for parents should include:

(1) An explanation of the nature of febrile convulsions, including information about the prevalence and prognosis.

(2) Instructions about the management of fever, the management of a convulsion, and the use of rectal diazepam.

(3) Reassurance.

This advice should be given verbally and a supplementary leaflet is helpful (see below).

\section{Advice to parents-Febrile convulsions}

Your child has had a febrile convulsion. We know it was a very frightening experience for you. You may have thought that your child was dead or dying, as many parents think when they first see a febrile convulsion. Febrile convulsions are not as serious as they appear.

\section{What is a febrile convulsion?}

It is an attack brought on by fever in a child aged between six months and five years.

\section{What is a convulsion?}

A convulsion is an attack in which the child becomes unconscious and usually stiff, with jerking of the arms and legs. It is caused by unusual electrical activity of the brain. The words convulsion, fit, and seizure have the same meaning.

\section{What shall I do if my child has another convulsion?}

Lay him on his side, with his head on the same level or slightly lower than the body. Note the time. Do not try to force anything into his mouth. Do not slap or shake the child.

The hospital may give you a medicine to insert into your child's bottom. This is called rectal diazepam. If the convulsion has not stopped by the time that you have found the tube, insert it into the child's bottom and express the contents of the tube. This treatment should stop the convulsion within 10 minutes. If it does not, take your child to the hospital. You may need to dial 999 to obtain an ambulance. Let your doctor know what has happened.

About one child in 30 will have had a febrile convulsion by the age of five years.

\section{Is it epilepsy?}

No. The word epilepsy is applied to fits without fever, usually in older children and adults.

\section{Do febrile convulsions lead to epilepsy?}

Rarely. 99 out of 100 children with febrile convulsions never have convulsions after they reach school age, and never have fits without fever.

\section{Do febrile convulsions cause permanent brain damage?}

Almost never. Very rarely a child who has a very prolonged febrile convulsion lasting half an hour or more may suffer permanent damage from it.

\section{What starts febrile convulsions?}

Any illness that causes a high temperature, usually a cold or other virus infection.

\section{Will it happen again?}

Three out of 10 children who have a febrile convulsion will have another one. The risk of having another febrile convulsion falls rapidly after the age of 3 years.

\section{Does the child suffer discomfort or pain during a convulsion?} No. The child is unconscious and unaware of what is happening.

\section{What shall I do if my child has fever?}

You can take the child's temperature by placing the bulb of the thermometer under his armpit for three minutes with his arm held against his side. Keep him cool by taking off his clothes and reducing the room temperature. Give plenty of fluids to drink. Give children's paracetamol medicine to reduce the temperature. The following doses should be given:

$\begin{array}{ll}\text { Up to } 1 \text { year old } & \text { one } 5 \mathrm{ml} \text { spoonful }(120 \mathrm{mg}) \\ \text { Aged } 1 \text { to } 3 \text { years } & \text { two } 5 \mathrm{ml} \text { spoonfuls }(240 \mathrm{mg})\end{array}$

Aged 4 years and over three $5 \mathrm{ml}$ spoonsfuls $(360 \mathrm{mg})$. Repeat the dose every four hours until the temperature falls to normal, and then every six hours for the next 24 hours.

If the child seems ill or has ear ache or sore throat, let your doctor see him in case any other treatment, such as an antibiotic, is needed. Antibiotics are not necesssary for most children with fever due to virus infections.

Is regular treatment with tablets or medicine necessary? Usually not. The doctor will explain to you if your child needs regular medicine.

H B Valman is consultant paediatrician, Northwick Park Hospital and Clinical Research Centre, Harrow, Middlesex

This is a chapter from the third edition of the $A B C$ of One to Seven, which will be published in September 1993. 